PROCEEDINGS OF THE

AMERICAN MATHEMATICAL SOCIETY

Volume 140, Number 3, March 2012, Pages 749-754

S 0002-9939(2011)10940-7

Article electronically published on June 23, 2011

\title{
A METRIC DISCREPANCY RESULT FOR LACUNARY SEQUENCES
}

\author{
KATUSI FUKUYAMA AND TETSUJIN WATADA
}

(Communicated by Richard C. Bradley)

\begin{abstract}
We prove that every value greater than or equal to $1 / 2$ can be a constant appearing in the law of the iterated logarithm for discrepancies of a lacunary sequence satisfying the Hadamard gap condition.
\end{abstract}

\section{INTRODUCTION}

In the theory of the uniform distribution, we use the following discrepancies of a sequence $\left\{a_{k}\right\}$ :

$$
\begin{aligned}
& D_{N}\left\{a_{k}\right\}=\sup _{0 \leq a^{\prime}<a<1}\left|\frac{1}{N} \#\left\{k \leq N \mid\left\langle a_{k}\right\rangle \in\left[a^{\prime}, a\right)\right\}-\left(a-a^{\prime}\right)\right|, \\
& D_{N}^{*}\left\{a_{k}\right\}=\sup _{0 \leq a<1}\left|\frac{1}{N} \#\left\{k \leq N \mid\left\langle a_{k}\right\rangle \in[0, a)\right\}-a\right|,
\end{aligned}
$$

where $\langle x\rangle$ denotes the fractional part $x-[x]$ of $x$.

One of the most typical results on the asymptotic behavior of discrepancies is celebrated in the Chung-Smirnov theorem, which asserts the law of the iterated logarithm for discrepancies of uniformly distributed i.i.d. $\left\{U_{k}\right\}$ :

$$
\varlimsup_{N \rightarrow \infty} \frac{N D_{N}^{*}\left\{U_{k}\right\}}{\sqrt{2 N \log \log N}}=\varlimsup_{N \rightarrow \infty} \frac{N D_{N}\left\{U_{k}\right\}}{\sqrt{2 N \log \log N}}=\frac{1}{2}, \quad \text { a.s. }
$$

We have similar phenomena without assuming the independence of the sequence of random variables. For a sequence $\left\{n_{k}\right\}$ satisfying the Hadamard gap condition,

$$
\inf _{k \in \mathbf{N}} n_{k+1} / n_{k}>1,
$$

Philipp [9, 10] proved the bounded law of the iterated logarithm

$$
\frac{1}{4 \sqrt{2}} \leq \Sigma^{*}\left\{n_{k} x\right\}:=\varlimsup_{N \rightarrow \infty} \frac{N D_{N}^{*}\left\{n_{k} x\right\}}{\sqrt{2 N \log \log N}} \leq \Sigma\left\{n_{k} x\right\}:=\varlimsup_{N \rightarrow \infty} \frac{N D_{N}\left\{n_{k} x\right\}}{\sqrt{2 N \log \log N}} \leq C
$$

for almost every $x$, where $C$ is a constant depending only on the infimum in (1.1). Recently it became possible to calculate concrete values of $\Sigma\left\{n_{k} x\right\}$ and $\Sigma^{*}\left\{n_{k} x\right\}$.

Received by the editors November 26, 2010 and, in revised form, December 8, 2010. 2010 Mathematics Subject Classification. Primary 11K38; Secondary 60F15.

Key words and phrases. Discrepancy, lacunary sequence, law of the iterated logarithm.

The first author was supported in part by KAKENHI 19204008.

(C)2011 American Mathematical Society Reverts to public domain 28 years from publication 
It is proved in [5] that for all real numbers $\theta>1$ there exists a constant $\Sigma_{\theta}$ such that

$$
\Sigma^{*}\left\{\theta^{k} x\right\}=\Sigma\left\{\theta^{k} x\right\}=\Sigma_{\theta}, \quad \text { a.e. }
$$

We have $\Sigma_{\theta}=1 / 2$ if $\theta$ satisfies the condition $\theta^{r} \notin \mathbf{Q}$ for all $r \in \mathbf{N}$. Otherwise let us express $\theta$ by $\theta=\sqrt[r]{p / q}$ where $r=\min \left\{n \in \mathbf{N} \mid \theta^{n} \in \mathbf{Q}\right\}$ and $\operatorname{gcd}(p, q)=1$. In this case $\Sigma_{\theta}$ does not depend on $r$ and is evaluated in the following cases:

$$
\Sigma_{\theta}= \begin{cases}\sqrt{(p q+1) /(p q-1)} / 2 & \text { if } p \text { and } q \text { are odd, } \\ \sqrt{(p+1) p(p-2) /(p-1)^{3}} / 2 & \text { if } p \geq 4 \text { is even and } q=1, \\ \sqrt{42} / 9 & \text { if } p=2 \text { and } q=1, \\ \sqrt{22} / 9 & \text { if } p=5 \text { and } q=2 .\end{cases}
$$

It is also proved that $\max _{\theta>1} \Sigma_{\theta}=\Sigma_{2}=\sqrt{42} / 9$ (cf. [7]). Aistleitner [1] gave a nearly optimal Diophantine condition on the sequence $\left\{n_{k}\right\}$ to have $\Sigma^{*}\left\{n_{k} x\right\}=$ $\Sigma\left\{n_{k} x\right\}=1 / 2$ a.e., which coincides with the case of uniformly distributed i.i.d.

In [8], it is proved that $\Sigma^{*}\left\{n_{k} x\right\}$ and $\Sigma\left\{n_{k} x\right\}$ are equal to a constant if $\left\{n_{k}\right\}$ is a subsequence of $\left\{\theta^{k}\right\}$ and the set of possible values of constants coincides with the interval $\left[1 / 2, \Sigma_{\theta}\right]$. Therefore every value in $[1 / 2, \sqrt{42} / 9]$ is proved to be a possible value of $\Sigma^{*}\left\{n_{k} x\right\}$ and $\Sigma\left\{n_{k} x\right\}$ for some $\left\{n_{k}\right\}$ satisfying the Hadamard gap condition.

It is natural to ask whether values greater than $\sqrt{42} / 9$ are also possible values of $\Sigma^{*}\left\{n_{k} x\right\}$ and $\Sigma\left\{n_{k} x\right\}$.

Related to this question, we can find the following result by Berkes and Philipp 3. For any real number $L$, there exists a sequence $\left\{n_{k}\right\}$ satisfying the Hadamard gap condition (1.1) such that $\Sigma\left\{n_{k} x\right\} \geq L$ a.e. Unfortunately, we cannot know by this result if $\Sigma\left\{n_{k} x\right\}$ is constant a.e. or not.

So far, the only known greater constants are those for the so-called HardyLittlewood-Pólya sequences defined as below: Let $q_{1}, \ldots, q_{\tau} \geq 2$ be relatively prime integers and let $\left\{n_{k}\right\}$ be an arrangement in increasing order of $\left\{q_{1}^{i_{1}} \ldots q_{\tau}^{i_{\tau}} \mid\right.$ $\left.i_{1}, \ldots, i_{\tau}=0,1,2, \ldots\right\}$. Then $\left\{n_{k}\right\}$ is said to be the Hardy-Littlewood-Pólya sequence generated by $q_{1}, \ldots, q_{\tau}$. As to this sequence, $\Sigma^{*}\left\{n_{k} x\right\}$ and $\Sigma\left\{n_{k} x\right\}$ are equal to a constant a.e. (cf. [6]), and when the set of generators consists of odd integers, then

$$
\Sigma^{*}\left\{n_{k} x\right\}=\Sigma\left\{n_{k} x\right\}=\frac{1}{2}\left(\prod_{i=1}^{\tau} \frac{q_{i}+1}{q_{i}-1}\right)^{1 / 2}, \quad \text { a.e. }
$$

See also 2 to find a detailed study for permutations of these sequences.

The last value becomes arbitrarily large if we choose generators properly. Unfortunately, when $\tau \geq 2$, Hardy-Littlewood-Pólya sequences do not satisfy the Hadamard gap condition. But the method of approximating the Hardy-LittlewoodPólya sequence by subsequences satisfying the Hadamard gap condition can be found in [4. We adopt this method together with randomization techniques to solve the above problem.

Now we are in a position to state our result.

Theorem 1.1. For all $\sigma \geq 1 / 2$, there exists a sequence $\left\{n_{k}\right\}$ of positive integers satisfying the Hadamard gap condition (1.1) such that

$$
\Sigma\left\{n_{k} x\right\}=\Sigma^{*}\left\{n_{k} x\right\}=\sigma, \text { a.e. }
$$


For $0<\sigma<1 / 2$, there exists a sequence $\left\{n_{k}\right\}$ with bounded gaps $n_{k+1}-n_{k}=$ $O(1)$ such that (1.3) holds (cf. [7]). It is an open question whether we can take such $\left\{n_{k}\right\}$ satisfying the Hadamard gap condition.

\section{ProOF}

We first note that discrepancies are written by

$$
D_{N}\left\{a_{k}\right\}=\sup _{0 \leq a<b<1}\left|\frac{1}{N} \sum_{k=1}^{N} \widetilde{\mathbf{1}}_{a, b}\left(a_{k}\right)\right|, \quad D_{N}^{*}\left\{a_{k}\right\}=\sup _{0 \leq a<1}\left|\frac{1}{N} \sum_{k=1}^{N} \widetilde{\mathbf{1}}_{0, a}\left(a_{k}\right)\right|,
$$

where $\widetilde{\mathbf{1}}_{a, b}(x)=\mathbf{1}_{[a, b)}(\langle x\rangle)-(b-a)$ and $\mathbf{1}_{[a, b)}$ denotes the indicator function of $[a, b)$.

Denote the sequence of all odd prime numbers by $3=q_{1}<q_{2}<\ldots$. Put $\phi(x)=x \vee 0$ and $\psi(x)=(-x) \vee 0$. We have $\phi(x)+\psi(x)=|x|$. Denote

$$
\Phi_{I ; j_{1}, \ldots, j_{\tau}}=\frac{\phi\left(I-\left|j_{1}\right|\right)}{I} \ldots \frac{\phi\left(I-\left|j_{\tau}\right|\right)}{I} \text { and } \Psi(x)=\sqrt{2 x \log \log x} .
$$

We use the next proposition.

Proposition 2.1. Let $0<p<1$ and let $\left\{X_{n}\right\}$ be a sequence of independent random variables defined on a probability space $(\Omega, \mathcal{F}, \mathbb{P})$ satisfying $\mathbb{P}\left(X_{i}=1\right)=1-\mathbb{P}\left(X_{i}=\right.$ $0)=p$. Let us regard the set $\left\{q_{\tau+1}^{i} \mid X_{i}(\omega)=1\right\}$ as an increasing sequence and denote it by $\left\{n_{k}^{\circ}(\omega)\right\}$. Then

$$
\mathbb{P}\left(\#\left\{n_{k}^{\circ}\right\}=\infty, \varlimsup_{N \rightarrow \infty} \Psi^{-1}(N)\left|\sum_{k=1}^{N} f\left(n_{k}^{\circ} x\right)\right|=\sigma(f, p) \text { a.e. } x, \text { for all } f \in B V_{0}\right)=1,
$$

where $B V_{0}$ denotes the class of functions $f$ of bounded variation with period 1 satisfying $\int_{0}^{1} f(t) d t=0$ and $\sigma^{2}(f, p)$ is given by

$$
\sigma^{2}(f, p)=\int_{0}^{1} f^{2}(x) d x+2 p \sum_{k=1}^{\infty} \int_{0}^{1} f\left(q_{\tau+1}^{k} x\right) f(x) d x .
$$

In 8 ] the authors proved a similar statement given by replacing 'for all $f \in B V_{0}$ ' with 'for all $\widetilde{\mathbf{1}}_{a, b}$ with $0 \leq a<b<1$ ' and $q_{\tau+1}$ with 3 . Proposition 2.1] can be proved completely in the same way. We apply it for

$$
f_{a, b}(x)=\frac{1}{I^{\tau / 2}} \sum_{i_{1}=0}^{I-1} \cdots \sum_{i_{\tau}=0}^{I-1} \widetilde{\mathbf{1}}_{a, b}\left(q_{1}^{i_{1}} \ldots q_{\tau}^{i_{\tau}} x\right) .
$$

Let us evaluate $\sigma^{2}\left(f_{a, b}, p\right)$. By changing the variable $y=q^{i \wedge i^{\prime}} x$ and by noting $i-i \wedge i^{\prime}=\phi\left(i-i^{\prime}\right)$ and $i^{\prime}-i \wedge i^{\prime}=\psi\left(i-i^{\prime}\right)$, we have

$$
\frac{1}{I} \sum_{i=0}^{I-1} \sum_{i^{\prime}=0}^{I-1} \int_{0}^{1} g\left(q^{\prime} q^{i} x\right) g\left(q^{\prime \prime} q^{i^{\prime}} x\right) d x=\sum_{j \in \mathbf{Z}} \frac{\phi(I-|j|)}{I} \int_{0}^{1} g\left(q^{\prime} q^{\phi(j)} y\right) g\left(q^{\prime \prime} q^{\psi(j)} y\right) d y
$$


Here we used the fact that $\#\left\{\left(i, i^{\prime}\right) \in\{0,1, \ldots, I-1\}^{2} \mid j=i-i^{\prime}\right\}=\phi(I-|j|)$. Hence we have

$$
\begin{aligned}
& \int_{0}^{1} f_{a, b}\left(q_{\tau+1}^{\phi(k)} x\right) f_{a, b}\left(q_{\tau+1}^{\psi(k)} x\right) d x \\
& =\frac{1}{I^{\tau}} \sum_{i_{1}, i_{1}^{\prime}=0}^{I-1} \ldots \sum_{i_{\tau}, i_{\tau}^{\prime}=0}^{I-1} \int_{0}^{1} \widetilde{\mathbf{1}}_{a, b}\left(q_{1}^{i_{1}} \ldots q_{\tau}^{i_{\tau}} q_{\tau+1}^{\phi(k)} x\right) \widetilde{\mathbf{1}}_{a, b}\left(q_{1}^{i_{1}^{\prime}} \ldots q_{\tau}^{i_{\tau}^{\prime}} q_{\tau+1}^{\psi(k)} x\right) d x \\
& =\sum_{j_{1}, \ldots, j_{\tau} \in \mathbf{Z}} \Phi_{I ; j_{1}, \ldots, j_{\tau}} \int_{0}^{1} \widetilde{\mathbf{1}}_{a, b}\left(q_{1}^{\phi\left(j_{1}\right)} \ldots q_{\tau}^{\phi\left(j_{\tau}\right)} q_{\tau+1}^{\phi(k)} x\right) \widetilde{\mathbf{1}}_{a, b}\left(q_{1}^{\psi\left(j_{1}\right)} \ldots q_{\tau}^{\psi\left(j_{\tau}\right)} q_{\tau+1}^{\psi(k)} x\right) d x .
\end{aligned}
$$

Therefore we have

$$
\begin{aligned}
& \sigma^{2}\left(f_{a, b}, 0\right)=\int_{0}^{1} f_{a, b}^{2}(x) d x \\
& =\sum_{j_{1}, \ldots, j_{\tau} \in \mathbf{Z}} \Phi_{I ; j_{1}, \ldots, j_{\tau}} \int_{0}^{1} \widetilde{\mathbf{1}}_{a, b}\left(q_{1}^{\phi\left(j_{1}\right)} \ldots q_{\tau}^{\phi\left(j_{\tau}\right)} x\right) \widetilde{\mathbf{1}}_{a, b}\left(q_{1}^{\psi\left(j_{1}\right)} \ldots q_{\tau}^{\psi\left(j_{\tau}\right)} x\right) d x,
\end{aligned}
$$

and by noting

$$
\sigma^{2}\left(f_{a, b}, 1\right)=\sum_{k \in \mathbf{Z}} \int_{0}^{1} f_{a, b}\left(q_{\tau+1}^{\phi(k)} x\right) f_{a, b}\left(q_{\tau+1}^{\psi(k)} x\right) d x
$$

we see that $\sigma^{2}\left(f_{a, b}, 1\right)$ equals

$$
\sum_{k, j_{1}, \ldots, j_{\tau} \in \mathbf{Z}} \Phi_{I ; j_{1}, \ldots, j_{\tau}} \int_{0}^{1} \widetilde{\mathbf{1}}_{a, b}\left(q_{1}^{\phi\left(j_{1}\right)} \ldots q_{\tau}^{\phi\left(j_{\tau}\right)} q_{\tau+1}^{\phi(k)} x\right) \widetilde{\mathbf{1}}_{a, b}\left(q_{1}^{\psi\left(j_{1}\right)} \ldots q_{\tau}^{\psi\left(j_{\tau}\right)} q_{\tau+1}^{\psi(k)} x\right) d x
$$

To evaluate the integrals above, we use the next lemma, which is proved in [5].

Lemma 2.2. For $x, y, \xi, \eta \in[0,1]$, put $V(x, \xi)=x \wedge \xi-x \xi$ and $\widetilde{V}(x, y, \xi, \eta)=$ $V(x, \xi)+V(y, \eta)-V(x, \eta)-V(y, \xi)$. For any positive integers $P$ and $Q$ with $\operatorname{gcd}(P, Q)=1$, we have

$$
\begin{gathered}
\int_{0}^{1} \widetilde{\mathbf{1}}_{a, b}(P x) \widetilde{\mathbf{1}}_{a, b}(Q x) d x=\frac{1}{P Q} \widetilde{V}(\langle P a\rangle,\langle P b\rangle,\langle Q a\rangle,\langle Q b\rangle), \\
\widetilde{V}(\langle P a\rangle,\langle P b\rangle,\langle Q a\rangle,\langle Q b\rangle) \leq V(\langle P(b-a)\rangle,\langle Q(b-a)\rangle) \leq 1 / 4 .
\end{gathered}
$$

If $P$ and $Q$ are odd and if $a=0, b=1 / 2$, then $\widetilde{V}(\langle P a\rangle,\langle P b\rangle,\langle Q a\rangle,\langle Q b\rangle)=1 / 4$.

By applying this lemma and by noting that $\phi(x)+\psi(x)=|x|$, we have

$$
\int_{0}^{1} \widetilde{\mathbf{1}}_{a, b}\left(q_{1}^{\phi\left(j_{1}\right)} \ldots q_{\tau}^{\phi\left(j_{\tau}\right)} q_{\tau+1}^{\phi(k)} x\right) \widetilde{\mathbf{1}}_{a, b}\left(q_{1}^{\psi\left(j_{1}\right)} \ldots q_{\tau}^{\psi\left(j_{\tau}\right)} q_{\tau+1}^{\psi(k)} x\right) d x \leq \frac{1}{4 q_{1}^{\left|j_{1}\right|} \ldots q_{\tau}^{\left|j_{\tau}\right|} q_{\tau+1}^{|k|}}
$$

and we see that the equality holds if $a=0$ and $b=1 / 2$. Put

$$
Q_{\tau}=\frac{1}{2}\left(\prod_{i=1}^{\tau} \frac{q_{i}+1}{q_{i}-1}\right)^{1 / 2} \quad \text { and } \quad Q_{\tau, I}=\frac{1}{2}\left(\sum_{j_{1}, \ldots, j_{\tau} \in \mathbf{Z}} \frac{\Phi_{I ; j_{1}, \ldots, j_{\tau}}}{q_{1}^{\left|j_{1}\right|} \ldots q_{\tau}^{\left|j_{\tau}\right|}}\right)^{1 / 2} .
$$


Hence we see

$$
\begin{aligned}
& \sigma^{2}\left(f_{a, b}, 0\right) \leq \frac{1}{4} \sum_{j_{1}, \ldots, j_{\tau} \in \mathbf{Z}} \frac{\Phi_{I ; j_{1}, \ldots, j_{\tau}}}{q_{1}^{\left|j_{1}\right|} \ldots q_{\tau}^{\left|j_{\tau}\right|}}=Q_{\tau, I}^{2}=\sigma^{2}\left(f_{0,1 / 2}, 0\right), \\
& \sigma^{2}\left(f_{a, b}, 1\right) \leq \frac{1}{4} \sum_{k, j_{1}, \ldots, j_{\tau} \in \mathbf{Z}} \frac{\Phi_{I ; j_{1}, \ldots, j_{\tau}}}{q_{1}^{\left|j_{1}\right|} \ldots q_{\tau}^{\left|j_{\tau}\right|} q_{\tau+1}^{|k|}}=Q_{\tau, I}^{2} \frac{q_{\tau+1}+1}{q_{\tau+1}-1}=\sigma^{2}\left(f_{0,1 / 2}, 1\right),
\end{aligned}
$$

which imply

$$
\begin{aligned}
& \sup _{S \ni a<b \in S} \sigma\left(f_{a, b}, 0\right)=\sup _{a \in S} \sigma\left(f_{0, a}, 0\right)=\sigma\left(f_{0,1 / 2}, 0\right)=Q_{\tau, I}, \\
& \sup _{S \ni a<b \in S} \sigma\left(f_{a, b}, 1\right)=\sup _{a \in S} \sigma\left(f_{0, a}, 1\right)=\sigma\left(f_{0,1 / 2}, 1\right)=Q_{\tau, I}\left(\frac{q_{\tau+1}+1}{q_{\tau+1}-1}\right)^{1 / 2}
\end{aligned}
$$

where $S=[0,1) \cap \mathbf{Q}$. By $\sigma^{2}\left(f_{a, b}, p\right)=(1-p) \sigma^{2}\left(f_{a, b}, 0\right)+p \sigma^{2}\left(f_{a, b}, 1\right)$, we also have

$$
\sup _{S \ni a<b \in S} \sigma\left(f_{a, b}, p\right)=\sup _{a \in S} \sigma\left(f_{0, a}, p\right)=\sigma\left(f_{0,1 / 2}, p\right)=\left(1-p+p \frac{q_{\tau+1}+1}{q_{\tau+1}-1}\right)^{1 / 2} Q_{\tau, I} \text {. }
$$

Let $\left\{n_{k}\right\}$ be an arrangement in increasing order of

$$
\left\{n_{k}^{\circ} q_{1}^{i_{1}} \ldots q_{\tau}^{i_{\tau}}: k=0,1, \ldots ; i_{1}, \ldots, i_{\tau}=0,1, \ldots, I-1\right\} .
$$

It is clear that $\left\{n_{k}\right\}$ satisfies the Hadamard gap condition. Let us take $\rho(k)$ such that $n_{\rho(k)}=n_{k}^{\circ} q_{1}^{I-1} \ldots q_{\tau}^{I-1}$. If $n_{k^{\prime}}^{\circ} q_{1}^{i_{1}^{\prime}} \ldots q_{\tau}^{i_{\tau}^{\prime}} \leq n_{k}^{\circ} q_{1}^{I-1} \ldots q_{\tau}^{I-1}$, then $n_{k^{\prime}}^{\circ} / n_{k}^{\circ} \leq$ $q_{1}^{I-1} \ldots q_{\tau}^{I-1}$. In case $k^{\prime} \geq k$, we have $3^{k^{\prime}-k} \leq n_{k^{\prime}}^{\circ} / n_{k}^{\circ}$ and thereby $k^{\prime} \leq k+$ $\log _{3} q_{1}^{I-1} \ldots q_{\tau}^{I-1}$. The last inequality is also valid when $k^{\prime}<k$. Hence we see

$$
\begin{aligned}
& \left\{n_{k}^{\circ} q_{1}^{i_{1}} \ldots q_{\tau}^{i_{\tau}} \mid k \leq K, i_{1}, \ldots, i_{\tau}<I\right\} \subset\left\{n_{k} \mid k \leq \rho(K)\right\} \\
& \quad \subset\left\{n_{k}^{\circ} q_{1}^{i_{1}} \ldots q_{\tau}^{i_{\tau}} \mid k \leq K+\log _{3} q_{1}^{I-1} \ldots q_{\tau}^{I-1}, i_{1}, \ldots, i_{\tau}<I\right\} .
\end{aligned}
$$

Therefore we have $K I^{\tau} \leq \rho(K) \leq K I^{\tau}+I^{\tau} \log _{3} q_{1}^{I-1} \ldots q_{\tau}^{I-1}$ and $\rho(K) \sim K I^{\tau}$.

For given $N$, take the largest $K$ such that $K I^{\tau} \leq N$. Then $N<K I^{\tau}+I^{\tau}$. It implies $|N-\rho(K)| \leq I^{\tau}+I^{\tau} \log _{3} q_{1}^{I-1} \ldots q_{\tau}^{I-1}$. Therefore we have

$$
\left|\sum_{k=1}^{N} \widetilde{\mathbf{1}}_{a, b}\left(n_{k} x\right)-\sum_{k=1}^{K} \sum_{i_{1}, \ldots, i_{\tau}<I} \widetilde{\mathbf{1}}_{a, b}\left(n_{k}^{\circ} q_{1}^{i_{1}} \ldots q_{\tau}^{i_{\tau}} x\right)\right|=O(1)
$$

and thereby we have

$$
\sigma\left(f_{a, b}, p\right)=\varlimsup_{K \rightarrow \infty} \Psi(K)^{-1} \sum_{k=1}^{K} f_{a, b}\left(n_{k}^{\circ} x\right)=\varlimsup_{N \rightarrow \infty} \Psi(N)^{-1}\left|\sum_{k=1}^{N} \widetilde{\mathbf{1}}_{a, b}\left(n_{k} x\right)\right|, \quad \text { a.e. }
$$

We use the fundamental relation below, which can be found in [7].

Lemma 2.3. For any countable dense subset $S$ of $[0,1)$ and for any sequence $\left\{n_{k}\right\}$ of positive real numbers satisfying the Hadamard gap condition, we have

$$
\begin{aligned}
& \varlimsup_{N \rightarrow \infty} \frac{N D_{N}\left\{n_{k} x\right\}}{\sqrt{2 N \log \log N}}=\sup _{S \ni a<b \in S} \varlimsup_{N \rightarrow \infty} \frac{1}{\sqrt{2 N \log \log N}}\left|\sum_{k=1}^{N} \widetilde{\mathbf{1}}_{a, b}\left(n_{k} x\right)\right|, \quad \text { a.e. }, \\
& \varlimsup_{N \rightarrow \infty} \frac{N D_{N}^{*}\left\{n_{k} x\right\}}{\sqrt{2 N \log \log N}}=\sup _{a \in S} \varlimsup_{N \rightarrow \infty} \frac{1}{\sqrt{2 N \log \log N}}\left|\sum_{k=1}^{N} \widetilde{\mathbf{1}}_{0, a}\left(n_{k} x\right)\right|, \quad \text { a.e. }
\end{aligned}
$$


By applying the lemma, we have

$$
\Sigma\left\{n_{k} x\right\}=\Sigma^{*}\left\{n_{k} x\right\}=\left(1-p+p \frac{q_{\tau+1}+1}{q_{\tau+1}-1}\right)^{1 / 2} Q_{\tau, I}, \quad \text { a.e. }
$$

Therefore, for every $\sigma \in\left(Q_{\tau, I},\left(\frac{q_{\tau+1}+1}{q_{\tau+1}-1}\right)^{1 / 2} Q_{\tau, I}\right)$, there exists a $p \in(0,1)$ such that (1.3) holds. Because $Q_{\tau, I}<Q_{\tau}, Q_{\tau, I} \uparrow Q_{\tau}$ as $I \rightarrow \infty$, and $\left(\frac{q_{\tau+1}+1}{q_{\tau+1}-1}\right)^{1 / 2} Q_{\tau}=$ $Q_{\tau+1}$, we see that for every $\sigma \in\left[Q_{\tau}, Q_{\tau+1}\right)$ there exists a sequence $\left\{n_{k}\right\}$ satisfying (1.1) and (1.3). Because $Q_{1}=1 / \sqrt{2}$ and $Q_{\tau} \rightarrow \infty$ as $\tau \rightarrow \infty$, we can conclude that for every $\sigma>1 / \sqrt{2}$ there exists a sequence $\left\{n_{k}\right\}$ satisfying (1.1) and (1.3). On the other hand, for any $\sigma \in[1 / 2,1 / \sqrt{2}]$ there exists a subsequence $\left\{n_{k}\right\}$ of $\left\{3^{k}\right\}$ such that (1.1) and (1.3) hold (cf. [8]).

\section{REFERENCES}

[1] C. Aistleitner, On the law of the iterated logarithm for the discrepancy of lacunary sequences, Trans. Amer. Math. Soc., 362 (2010) 5967-5982. MR2661504

[2] C. Aistleitner, I. Berkes, R. Tichy, On permutation of Hardy-Littlewood-Polya sequences, Trans. Amer. Math. Soc. (to appear).

[3] I. Berkes, W. Philipp, The size of trigonometric and Walsh series and uniform distribution mod 1, Jour. London Math. Soc. (2), 50 (1994) 454-464. MR1299450 (96e:11099)

[4] K. Fukuyama, An asymptotic property of gap series, Acta Math. Hungar., 97 (2002) 257-264. MR:1933732 (2003i:42013)

[5] K. Fukuyama, The law of the iterated logarithm for discrepancies of $\left\{\theta^{n} x\right\}$, Acta Math. Hungar., 118 (2008) 155-170. MR2378547 (2008m:60049)

[6] K. Fukuyama, K. Nakata, A metric discrepancy result for the Hardy-Littlewood-Pólya sequences, Monatsh. Math., 160 (2010) 41-49. MR2610311

[7] K. Fukuyama, A central limit theorem and a metric discrepancy result for sequence with bounded gaps, Dependence in Probability, Analysis and Number Theory, A volume in memory of Walter Philipp, Kendrick Press, Heber City, UT (2010), 233-246.

[8] K. Fukuyama, N. Hiroshima, Metric discrepancy results for subsequences of $\left\{\theta^{k} x\right\}$, Monatsh. Math. (to appear).

[9] W. Philipp, Limit theorems for lacunary series and uniform distribution mod 1, Acta Arith., 26 (1975) 241-251. MR0379420(52:325)

[10] W. Philipp, A functional law of the iterated logarithm for empirical distribution functions of weakly dependent random variables, Ann. Probab., 5 (1977) 319-350. MR0443024 (56:1397)

Department of Mathematics, Kobe University, Rokko, Kobe, 657-8501 Japan

E-mail address: fukuyama@math.kobe-u.ac.jp

Department of Mathematics, Kobe University, Rokko, Kobe, 657-8501 Japan 